\title{
An case report of persistent giardiasis
}

\author{
Daniele Crotti*, Elisabetta Antonelli^, Maria Letizia D’Annibale ${ }^{\circ}$ \\ * Libero Professionista in Parassitologia e Microbiologia Clinica, Perugia, Italy \\ ^ U. O. di Gastroenterologia, Azienda Ospedaliera di Perugia,Italy \\ - S. C. di Microbiologia, Azienda Ospedaliera di Perugia, Italy
}

Key Words: epidemiology, Giardia duodenalis, giardiasis, stool parasitological diagnosis, zoonoses

\section{Giardiasi autoctona: descrizione di un caso ostinato}

\section{SUMMARY}

The authors describe a persistent case of autoctonous giardiasis in an Italian adult man. Patient's habits, clinical findings and diagnostic criteria are reported.The persistent elimination of Giardia spp. cysts with faeces along several month and years could be related to a repeated environmental ingestion or due to the onset of resistance towards the antiprotozoal drug.

Received January I2, 2009

Accepted February 18, 2009

\section{INTRODUZIONE}

Giardia duodenalis, sinonimo di Giardia intestinalis e di Giardia lamblia, è tuttora un protozoo largamente diffuso in ogni dove, specialmente nei climi tropicali e sub-tropicali dei cinque continenti del nostro globo $(9,10,14)$. E' tuttavia presente anche nelle regioni a clima temperato, essendo così reperibile anche in Italia, a prevalenze peraltro più basse e comunque variabili $(2,3,12)$. A trasmissione fecale-orale, tipicamente o comunque preferibilmente veicolata dall'acqua, questo flagellato riconosce nello stadio di cisti la forma infettante e di sopravvivenza, mentre il trofozoite, escistato nel duodeno ove usualmente si localizza, è la forma responsabile della malattia intestinale $(2,12,14)$. I trofozoiti, discesi a livello del grosso intestino con il transito intestinale, si trasformano in cisti che vengono eliminate nell'ambiente esterno ove possono sopravvivere anche a lungo $(4,10,14)$. L'ingestione accidentale con acque contaminate o con cibi a loro volta contaminati da acque infette perpetua la catena di tale infezione, sebbene siano riconosciute altre forme di infezione, come quella uomo-uomo o animale-uomo (1, $4,13)$.

Da un punto di vista epidemiologico ed epizootico va infatti precisato che anche svariati animali, in particolar modo alcuni mammiferi (soprattutto certi roditori), possono albergare tale protozoo, che pertanto può essere considerato responsabile di una tipica zoonosi, sebbene sembra altrettanto verosimile come nei singoli generi animali tale protozoo si possa essere adattato, per cui potrebbe essere altresì giustificata una diversa speciazione per lo stesso genere Giardia; così: G. bovis nel bovino, G. cati nel gatto e G. canis nel cane, $C$. muris nei roditori (C. chinchillae nei cincillà, nella fattispecie), C. agilis negli anfibi, C. psittaci e $C$. ardae in svariati uccelli, essendo invece $G$. duodenalis tipica dell'uomo $(4,14)$. A tale fine ricerche approfondite di tipo genetico sono in corso da più gruppi di ricercatori proprio per definire tale stato di cose, ovverosia se la giardiasi sia o no una zoonosi e/o se la giardiasi possa essere una zoonosi e al contempo essere genere-specifica; in altre parole se si tratti di assemblaggi diversificati di G. duodenalis, o di genotipi diversi di $G$. duodenalis, o di specie diverse di Giardia, ovvero specie genere-specifiche di Giardia $(1,7,8,12$, 14).

Nell'uomo G. duodenalis può essere responsabile di sintomatologia variabile: da situazioni in cui è presente una sintomatologia più o meno sfumata e in ogni caso aspecifica (ove sovente il dolore addominale saltuario è il disturbo prevalente) a situazioni in cui la giardiasi si presenta con diarrea acuta (talora con emissione anche di muco) o con diarrea protratta nel tempo, mentre altre volte la giardiasi è pressoché asintomatica $(1,4,12$, 14). Al proposito il dilemma è, anche qui, se si

\section{Corresponding author: Daniele Crotti}

Libero Professionista in Parassitologia e Microbiologia Medica,

Strada Comunale per Pilonico Paterno 4, 06 I 34 Pianello, Perugia, Italy - Telefono: 075602372

e-mail: nenedc@tin.it 
tratta sempre e soltanto di G. duodenalis o non vi sono invece specie di versificate ( $G$. duodenalis, G. intestinalis, G. lamblia), oppure, più verosimilmente, non si tratti di assemblaggi genici o genotipi diversi correlati o correlabili alle differenti situazioni cliniche $(5,7,8)$.

Sono tutti aspetti in fase di studio, di valutazione e di approfondimento, per meglio comprendere sia l'epidemiolgoia sia la clinica e, con quest'ultima, la patogenicità di tale protozoo $(1,7)$. Ultimo aspetto di attualità in tema di Giardia spp. e di giardiasi, è anche quello che riguarda la potenziale o la possibile insorgenza di resistenze ai farmaci, in particolar modo al farmaco usualmente d'elezione per la sua eradicazione, ossia il Metronidazolo (1, 11).

Indipendentemente da tali aspetti, in tale comunicazione breve si vuole presentare un caso forse insolito di giardiasi, o che, comunque, può riflettere parte delle problematiche sopra elencate, e, nella fattispecie, sottolineare come $G$. duodenalis sia non così raramente osservabile, proprio nella popolazione autoctona nel nostro paese $(2,3)$.

\section{DESCRIZIONE DEL CASO}

In data 12 gennaio 2005 si presenta al Day Hospital di un reparto di Medicina Interna un soggetto di sesso maschile, di anni 57, per essere sottoposto a visita specialistica e indagini relative in quanto affetto da tempo da un fastidioso prurito al dorso e agli arti accompagnato da vaghi disturbi intestinali non specifici. Null'altro emerge dalla visita clinica; in ogni caso vengono richieste indagini emato-chimiche ed indagini copro-parassitologiche. L'esame emocromocitometrico risulta nella norma, così come il profilo biochimico e immunologico. Il soggetto viene dimesso con la diagnosi di prurito generalizzato di n.d.d.

Nella scheda di accompagnamento dei 3 campioni fecali inviati per l'esame copro-parassitologico standard (ECPS) viene segnalata la presenza di una giardiasi quattro anni prima. L'ECPS viene eseguito con osservazione microscopica diretta, osservazione microscopica dopo arricchimento formolo-etilacetato, osservazione microscopica dopo colorazione di Giemsa (2, 3). Vengono rilevate numerose cisti di $G$. duodenalis già all'osservazione diretta condotta in soluzione fisiologica ed in soluzione iodata. In conseguenza di ciò al soggetto viene praticata ambulatorialmente una colonscopia, che mette in evidenza la presenza di in piccolo polipo in sede intestinale. Infine gli viene prescritta una terapia con Metronidazolo per cinque giorni.

A distanza di alcuni giorni dalla fine della terapia orale allo stesso soggetto viene richiesto un ECPS di controllo. Questo viene eseguito tra il 6 ed il 9 febbraio e tutti e 3 i campioni fecali risultano negativi per strutture parassitarie.

In data 7 marzo il soggetto viene ricoverato presso il Reparto di Gastroenterologia ed Epatologia per essere sottoposto alla resezione endoscopica del polipo. Prima di procedere si vuole peraltro escludere la presenza di patogeni intestinali, per cui viene richiesta una copro coltura (che risulterà negativa per Salmonella, Shigella e Campylobacter) e un ECPS. Già dai primi due campioni di feci, dell'8 marzo, si evidenzia la ricomparsa di cisti di $G$. duodenalis. In data 9 marzo al paziente viene raccolto, mediante aspirazione, il succo duodenale che viene subito inviato al laboratorio parassitologico per le indagini di competenza. L'osservazione microscopica mette in evidenza come atteso la presenza di trofozoiti di Giardia duodenalis, che vengono poi colorati con il Giemsa per una adeguata conservazione (Figura I). Al paziente viene prescritta una terapia specifica sempre con Metronidazolo (1 compressa da $250 \mathrm{mg}$ 4/dì) ma per dieci giorni. Viene dimesso e lo si invita a ricontrollarsi una settimana dopo la fine della terapia. Il 29 marzo vengono così analizzati i primi 3 campioni fecali di controllo, che risultano negativi per Giardia spp. Su invito specifico del responsabile laboratorista vengono però immediatamente richiesti altri 3 campioni fecali da raccogliersi preferibilmente a giorni alternati $(2,3,6)$. In data 4 aprile viene consegnato un campione di feci che risulta positivo sempre per cisti di G. duodenalis. Il paziente si ripresenta soltanto verso la fine di aprile per ritirare l'esito del quarto campione fecale successivo alla fine del II ciclo terapeutico e in tale circostanza viene sottoposto ad un ulteriore e più ampia indagine anamnestica. Il soggetto abita in zona rurale e alleva per diletto alcuni capi di ovini ed equini, e possiede sia un cane che alcuni gatti. L'area è sita vicino alla abitazione; abitazione nella quale vive

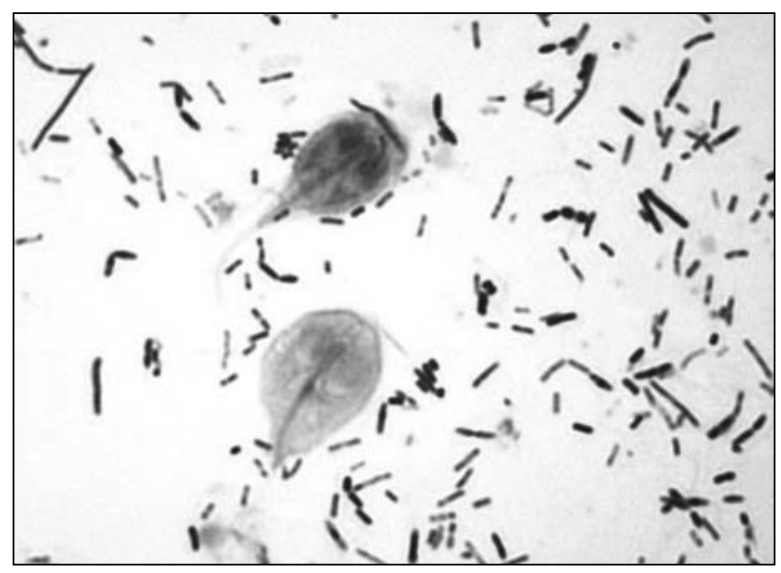

Figura I. Osservazione microscopica. Presenza di trofozoiti di Giardia duodenalis, colorati con il Giemsa per una adeguata conservazione 
con la madre, mentre i due figli, di cui uno sposato con bambino piccolo, abitano negli appartamenti sopra e sottostanti. Risulterebbe che nel recente passato il nipotino abbia sofferto di ripetuti episodi diarroici (mai indagati a dovere). L'area dove alleva i capi di bestiame di cui sopra è fornita di un pozzo d'acqua non controllato, cui peraltro il soggetto dice di non accedere personalmente, né per berne l'acqua né per utilizzarla ad uso alimentare in casa, ma soltanto per usarla a scopo irriguo e zootecnico.

Il soggetto conferma di aver fatto in modo esaustivo la terapia prescritta, ma è piuttosto vago e reticente circa le informazioni riguardante il nucleo famigliare allargato. Si riesce soltanto a farsi portare, in data 29 aprile, 2 campioni fecali della madre, 1 campione di feci rispettivamente del cane, di un ovino e di un equino, oltre ad altri 3 campioni personali. Mentre i 3 campioni del soggetto sono sempre positivi per G. duodenalis, i campioni della madre e degli animali sono negativi.

Al paziente viene allora suggerita una terapia rappresentata da una unica dose di 3 grammi di Tinidazolo e a seguire sette giorni di Metronidazolo (250 mg 3/dìe), ipotizzando vuoi la possibilità di reinfezione accidentale idro-alimentare vuoi la comparsa di una resistenza al farmaco d'elezione.

Dopo circa 1 anno, in data 2 febbraio 2006, il soggetto porterà 3 campioni di feci che risulteranno positivi per cisti di G. duodenalis; in data 19 marzo 2009 l'analisi del succo duodenale sarà positivo per trofozoiti di G. duodenalis.

Il soggetto non si è poi più ripresentato per eventuali controlli o terapie o altro ancora.

\section{BIBLIOGRAFIA}

1. Adam RD. Biology of Giardia lamblia. CMR 2001; 14: 447-475

2. Bernieri F, Crotti D. Infezioni da protozoi. Microbiol Med 2001; 16: 98-108

3. Crotti D, D’Annibale ML. Attualità in tema di infezioni intestinali causate da Dientameoba fragilis e Giardia duodenalis. Rec Prog Med 2007; 98: 361-366

4. Faust EC, Russell PF, Jung RC. Craig and Faust's Clinical Parasitology. Lea \& Febiger eds, Philadelphia, 1970, VIII ed.

5. Garcia LS. Practical Guide To Diagnostic Parasitology. ASM Press, Washington, D. C., 1999

6. Hanson KL, Cartwright CP. Use o fan Enzyme Immunoassay Does Not Eliminate the Need To Analyze Multiple Stool Specimens for Sensitive Detection of Giardia lamblia. JCM 2001; 39: 474-477

7. Lalle M, Pozio E, Capelli G, et al. Genetic heterogeneity at the ß-giardin locus among human and animal isolates of Giardia duodenalis and identification of potentially zoonotic subgenotypes. Internat $J$ Parasitol 2005, 35: 207-213

8. Lalle M, Pozio E, Capelli G, et al. Giardia duodenalis isolates from humans, dogs and calves: molecular genotyping and the role of zoonotic transmission. Parassitologia 2004, 46 (Suppl 1): 176

9. Lora-Suarez F, Marin-Vasquez C, Loango $\mathrm{N}$, et al. Giardiasis in children living in post-earthquacke camps from Armenia (Colombia). BMC Public Health 2002, 2: 5

10. Minvielle MC, Pezzani BC, Cordoba MA, et al. Epidemiological survey of Giardia spp. and Blastocystis hominis in an Argentinian rural community. Kor J Parassitol 2004, 42: 121 - 127

11. Özbilgin A, Ertan P, Yereli K et al. Giardiasis treatment in Turkish Children with a Single Dose of ornidazole. Scand J Infec Dis 2002, 34: 918-920

12. Pampiglione S, Canestri Trotti G. Guida allo studio della Parassitologia. Società Editrice Esculapio, Bologna, III ed., 1999

13. Stuart JM, Orr HJ, Warburton FG et al. Risk Factors for Sporadic Giardiasis: A Case-Control Study in Southwestern England. EID 2003, 9: 229-233

14. Thompson RCA. Giardia infections, in: Zoonoses, SR Palmer, Lord Soulsby and DIH Simpson eds, Oxford University Press, 1998, New York 